\title{
Magnetic loop emergence within a granule
}

\author{
P. Gömöry ${ }^{1,4}$, C. Beck ${ }^{2}$, H. Balthasar ${ }^{3}$, J. Rybák ${ }^{4}$, A. Kučera ${ }^{4}$, J. Koza ${ }^{4}$, and H. Wöhl ${ }^{5}$
}

\author{
1 IGAM/Kanzelhöhe Observatory, Institute of Physics, Universität Graz, Universitätsplatz 5, 8010 Graz, Austria \\ 2 Instituto de Astrofísica de Canarias (IAC), via Láctea, 38205 La Laguna, Spain \\ Astrophysikalisches Institut Potsdam, An der Sternwarte 16, 14482 Potsdam, Germany \\ 4 Astronomical Institute, Slovak Academy of Sciences, 05960 Tatranská Lomnica, Slovakia \\ e-mail: gomory@astro.sk \\ 5 Kiepenheuer-Institut für Sonnenphysik, Schöneckstr. 6, 79104 Freiburg, Germany
}

Received 1 July 2009 / Accepted 29 August 2009

\section{ABSTRACT}

\begin{abstract}
Aims. We investigate the temporal evolution of magnetic flux emerging within a granule in the quiet-Sun internetwork at disk center. Methods. We combined IR spectropolarimetry of high angular resolution performed in two Fe I lines at $1565 \mathrm{~nm}$ with specklereconstructed G-band imaging. We determined the magnetic field parameters by a LTE inversion of the full Stokes vector using the SIR code, and followed their evolution in time. To interpret the observations, we created a geometrical model of a rising loop in 3D. The relevant parameters of the loop were matched to the observations where possible. We then synthesized spectra from the 3D model for a comparison to the observations.

Results. We found signatures of magnetic flux emergence within a growing granule. In the early phases, a horizontal magnetic field with a distinct linear polarization signal dominated the emerging flux. Later on, two patches of opposite circular polarization signal appeared symmetrically on either side of the linear polarization patch, indicating a small loop-like structure. The mean magnetic flux density of this loop was roughly $450 \mathrm{G}$, with a total magnetic flux of around $3 \times 10^{17} \mathrm{Mx}$. During the $\sim 12$ min episode of loop occurrence, the spatial extent of the loop increased from about 1 to 2 arcsec. The middle part of the appearing feature was blueshifted during its occurrence, supporting the scenario of an emerging loop. There is also clear evidence for the interaction of one loop footpoint with a preexisting magnetic structure of opposite polarity. The temporal evolution of the observed spectra is reproduced to first order by the spectra derived from the geometrical model. During the phase of clearest visibility of the loop in the observations, the observed and synthetic spectra match quantitatively.

Conclusions. The observed event can be explained as a case of flux emergence in the shape of a small-scale loop. The fast disappearance of the loop at the end could possibly be due to magnetic reconnection.
\end{abstract}

Key words. Sun: photosphere - magnetic fields - Sun: granulation

\section{Introduction}

Our knowledge of small-scale magnetic fields located in the photosphere and low chromosphere has evolved extremely fast thanks to the high spatial resolution observations done with the Hinode satellite (e.g., Lites et al. 2008), and the improvement of the spatial resolution of large ground-based solar telescopes achieved by adaptive optics systems (e.g., von der Lühe et al. 2003). The results of such observations tracing the weakest magnetic fields can now be compared directly with theoretical models, for example on the question whether a local dynamo operates in the solar photosphere (Cattaneo 1999). The observations may also help to refine modern numerical simulations of surface magnetoconvection (Vögler et al. 2005; Schaffenberger et al. 2006; Stein \& Nordlund 2006; Abbett 2007) by providing more stringent boundary conditions on magnetic field properties in the solar photosphere.

Direct measurements of the 3D topology and the temporal evolution of magnetic loops associated with small granular and intergranular structures are, however, still rare. The measurements require accurate spectropolarimetric 2D data of all Stokes parameters with high spatial resolution, high signal-to-noise ratio, and high cadence. Martínez González et al. (2007) reported observations of the magnetic field vector in the internetwork. They showed evidence that small-scale (only $2-6$ arcsec long) low-lying loops connect at least $10-12 \%$ of the internetwork magnetic flux measured at $1565 \mathrm{~nm}$.

Centeno et al. (2007, CE07) and Orozco Suárez et al. (2008, OR08) analyzed the temporal evolution of such small-scale magnetic features. They used similar data sets of the Fe I $630.15 \mathrm{~nm}$ and $630.25 \mathrm{~nm}$ spectral lines from the Hinode/SOT spectropolarimeter (Lites et al. 2001; Kosugi et al. 2007). OR08 constructed maps of the temporal evolution of linear and circular polarization signals, line-of-sight velocity, and the intensities of the Fe I $630.25 \mathrm{~nm}$ and CaII H spectral line. They found magnetic signals appearing at the central parts of granules, but without a significant linear polarization signal. Estimated lifetimes of the events were 20 and $14 \mathrm{~min}$. They did not find any significant coupling of the events to the chromosphere. CE07 applied an inversion assuming local thermodynamic equilibrium (LTE) to the Hinode spectra to retrieve information on the temporal evolution of the magnetic flux and its topology. Similar to OR08, they also reported the appearance of magnetic signals in the central parts of granules, and documented a drift of vertical dipoles toward the surrounding intergranular lanes. CE07 found a slightly shorter lifetime of only $8 \mathrm{~min}$. Moreover, they found that the appearance of horizontal magnetic fields precedes that of the vertical fields. They interpreted their results as the rise of small-scale magnetic loops through the photosphere, although they could not exclude a descending loop. 
In this paper, we present a similar analysis of the temporal evolution of the appearance and topology changes during a particular flux emergence event in the internetwork at the disk center, using ground-based observations in the infrared spectral region rendering high polarimetric precision and magnetic sensitivity. We compare these observations with synthetic spectra from a geometrical model of a rising loop to investigate whether the observations can be interpreted in such terms.

\section{Observations}

We observed a very quiet region at disk center using the German Vacuum Tower Telescope (VTT, Schröter et al. 1985) located at the Observatorio del Teide (Tenerife, Spain) on June 6, 2008 between 09:57:50-11:10:30 UT.

We used the Kiepenheuer-Institut Adaptive Optics System (KAOS, Berkefeld 2006) to improve the spatial resolution by a real-time correction of wavefront distortions. The uncorrected Fried parameter $r_{0}$ reached an average value of about $9 \mathrm{~cm}$ during the whole observing run that is sufficient to reach the diffraction limit of the VTT of around 0.' 6 at infrared (IR) wavelengths after the correction.

The light coming from the telescope was separated into visible and IR radiation components using a dichroic beam splitter (splitting edge at $800 \mathrm{~nm}$ ). The IR radiation was sent to the Tenerife Infrared Polarimeter (TIP 2, Collados et al. 2007) attached to the main spectrograph of the VTT. The visible fraction of the radiation was reflected to an optical bench where the following instruments were fed in parallel by two additional achromatic beam splitters: a G-band broad-band filter (center and $F W H M: 430.6 \mathrm{~nm}, 0.9 \mathrm{~nm}$ ), a narrow-band Zeiss Lyot filter for $\mathrm{H} \alpha(F W H M \sim 0.025 \mathrm{~nm})$, and the TESOS 2D spectrometer (Kentischer et al. 1998; Tritschler et al. 2002). More information on the instrumental setup can be found also in Kučera et al. (2008) or Beck et al. (2007).

We used the TIP spectropolarimeter with a 0.45 wide and $77^{\prime \prime}$ long slit to repeatedly scan a narrow $5^{\prime \prime}$ wide area in 10 steps. TIP covered the spectral region around the Fe I lines at $1564.85 \mathrm{~nm}$ and $1565.28 \mathrm{~nm}$. For a more detailed description of these lines see, e.g., Bellot Rubio et al. (2004) or Khomenko \& Collados (2007). Spatial and spectral sampling of the data were $0{ }^{\prime} 18 / \mathrm{px}$ and $14.64 \mathrm{m \AA} / \mathrm{px}$, respectively. We acquired the four modulation states employed by TIP with $250 \mathrm{~ms}$ exposures each. To obtain a higher signal-to-noise ratio in the recorded Stokes parameters $(I, Q, U, V)$, we then added 8 sets of the modulation states at each scan position, yielding a total integration time of $8 \mathrm{~s}$ and a cadence of $\sim 10 \mathrm{~s}$ for two consecutive scanning positions. The acquisition of the whole 10-step raster thus took about $100 \mathrm{~s}$ for every repetition of the scanning.

The G-band images were acquired with a 14-bit PCO 4000 digital camera. We set the exposure time to $5 \mathrm{~ms}$ and the cadence to $0.6 \mathrm{~s}$; the field of view (FoV) was $70^{\prime \prime} \times 100^{\prime \prime}$, and the spatial sampling was $0{ }^{\prime} 08$ per pixel. The $\mathrm{H} \alpha$ data (see, e.g., Kučera et al. 2008) and the data taken with the TESOS instrument were not used for this publication.

\section{Data reduction and analysis}

We photometrically corrected all data by subtracting the dark current and applying flat-field frames. To compensate for the instrumental polarization, we applied the correction described by Schlichenmaier \& Collados (2002) to the spectra of TIP, using a data set obtained with the IR instrument calibration unit

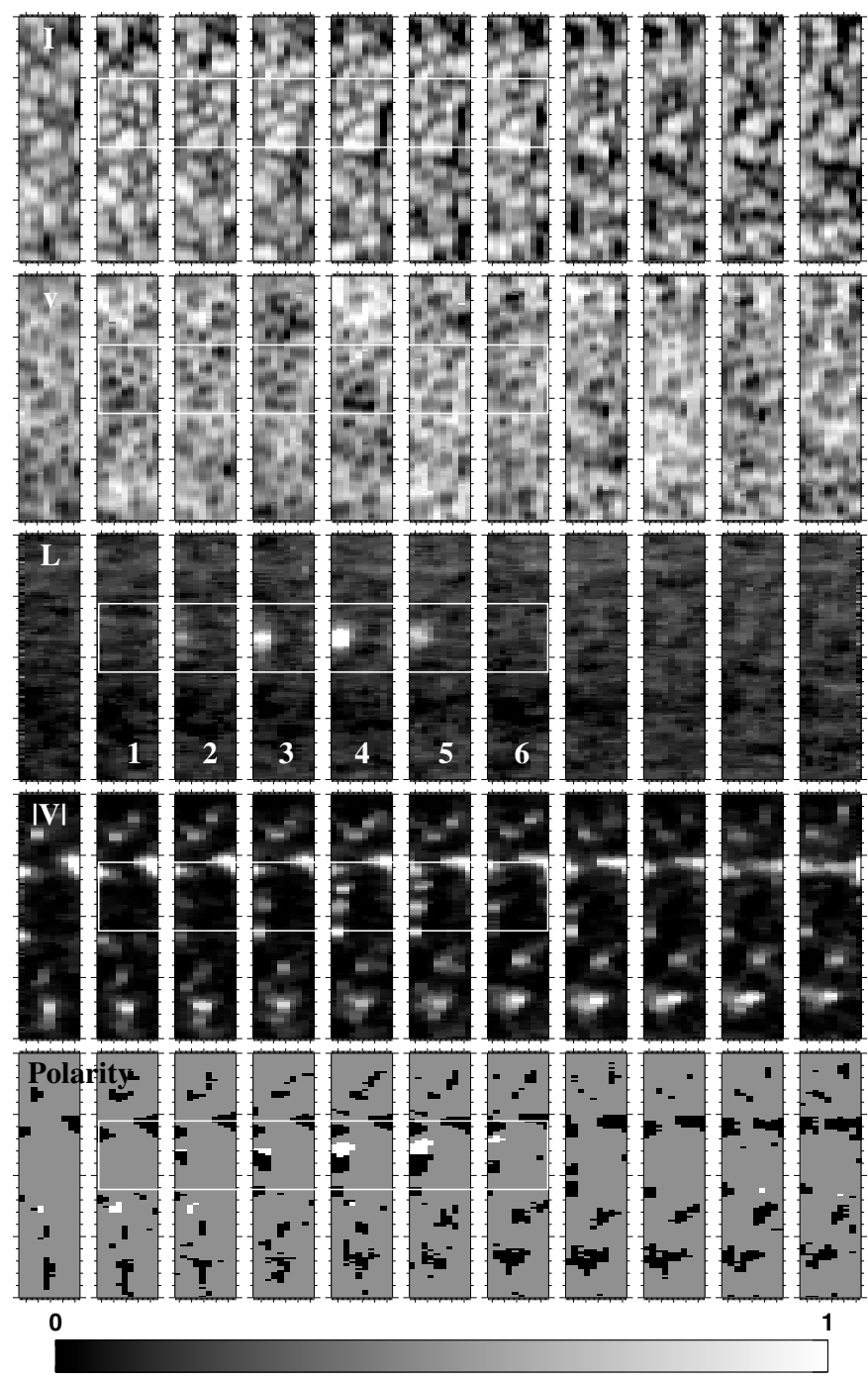

I: $0.96-1.04 ; \mathrm{v}:-1.1-1.1$ [km/s]; L: $12 \times 10^{-4}-14.5 \times 10^{-4}$; IVI: $9 \times 10^{-4}-15.5 \times 10^{-4}$

Fig. 1. Overview of the TIP dataset. The panels show from top to bottom 2D maps of the continuum intensity, the Doppler shifts of the Fe I $1564.85 \mathrm{~nm}$ line (black to mid-gray colors correspond to the blueshifts, mid-gray to white colors represent the redshifts), the net linear polarization, the circular polarization, and the polarity of the detected magnetic structures. The size of a single map here is $5^{\prime \prime} \times 20^{\prime \prime}$; their corresponding positions inside the G-band FoV are marked in Fig. 3.

of the VTT. Then, for a complete correction, the coelostat configuration of the telescope was modeled according to Collados (1999) and Beck et al. (2005). We finally estimated statistically the residual crosstalk following Collados (2003). An overview of the relevant section of the dataset obtained with TIP is shown in Fig. 1. It shows from top to bottom the continuum intensity $I_{\mathrm{c}}$, the line-core Doppler shifts of the Fe I $1564.8 \mathrm{~nm}$ line, the total linear polarization $L=\int\left(Q^{2}+U^{2}\right)^{1 / 2} / I_{\mathrm{c}} \mathrm{d} \lambda$, the total circular polarization $|V|=\int|V| / I_{\mathrm{c}} \mathrm{d} \lambda$, and the magnetic field polarity derived from the order of minimum and maximum of the $V$ signal. The region of the flux emergence is marked with white rectangles; the repetitions of the scanning marked with the consecutive numbers 1 to 6 are used for comparison with the geometrical loop model later on (see also Fig. 4).

To derive the magnetic field parameters, we used the SIR code (Stokes Inversion based on Response functions, Ruiz Cobo $\&$ del Toro Iniesta 1992) for a simultaneous inversion of the 
P. Gömöry et al.: Magnetic loop emergence within a granule
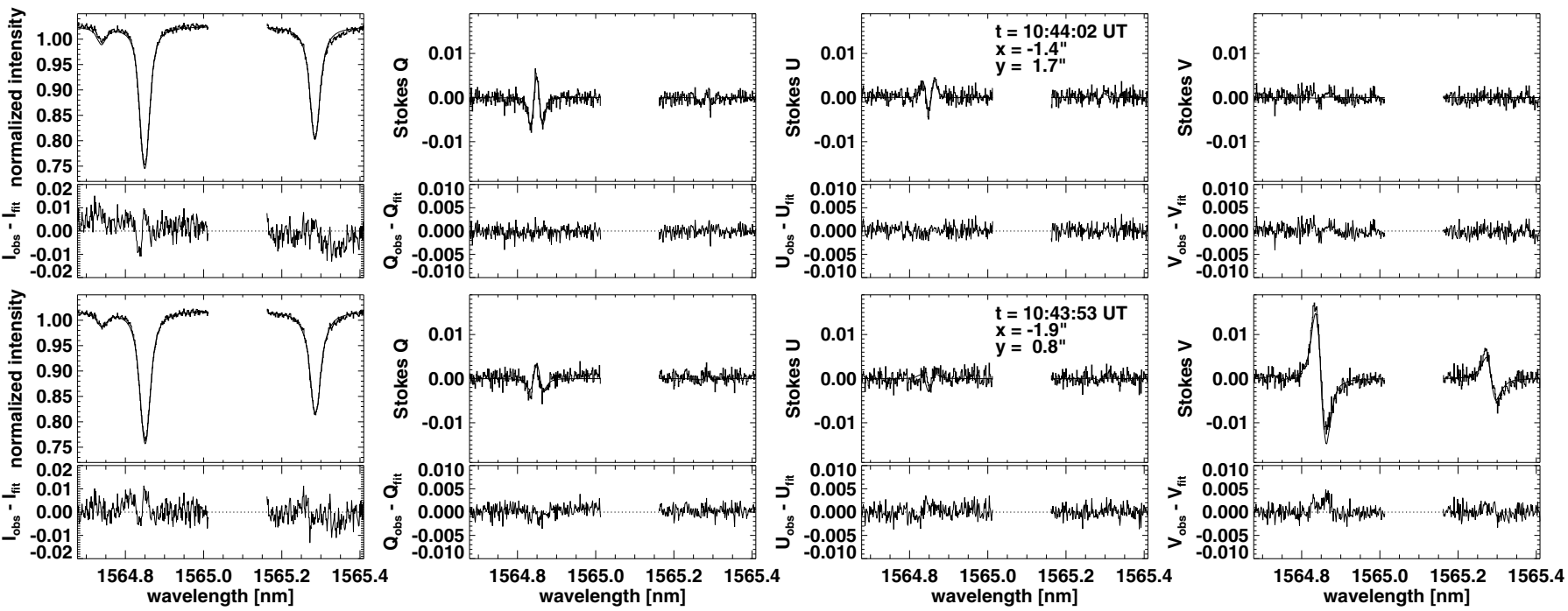

Fig. 2. Examples of the typical Stokes profiles detected in the middle part (top row) and southern footpoint (bottom row) of the emerging magnetic feature. Thin lines show observed profiles, thick lines the best-fit profiles of the inversion. Narrow panels show their residual differences. The $x, y$, $t$ parameters represent spatial positions of extracted profiles and the times of particular exposures.

Stokes profiles of Fe I lines at $1565 \mathrm{~nm}$ in a similar way as described by Bellot Rubio et al. (2004). We used a two-component model of the solar atmosphere with a magnetic and a field-free component that allows us to derive accurate parameters also in the case of spatially unresolved magnetic fields. All magnetic parameters were kept constant with height during the inversion. To demonstrate the quality of the performed inversion, we display the typical Stokes profiles detected in the middle part and in the southern footpoint of the emerging feature in Fig. 2 together with the corresponding best-fit profiles determined by SIR, and their residual differences. The rms noise in continuum windows of the observed Stokes profiles shown in Fig. 2 was $3.5 \times 10^{-3}$, $1.1 \times 10^{-3}, 1.1 \times 10^{-3}$, and $1 \times 10^{-3}$ for the middle part and $3.7 \times 10^{-3}, 1.1 \times 10^{-3}, 1.5 \times 10^{-3}$, and $8 \times 10^{-4}$ for the southern footpoint in the $I, Q, U$, and $V$ Stokes profiles, respectively.

We used the Kiepenheuer-Institut Speckle Interferometry Package (KISIP) code (Wöger et al. 2008) for the speckle reconstruction of the G-band data. A burst of 100 images was selected for each reconstruction leading to a temporal cadence of $63 \mathrm{~s}$. The maximal time difference between a TIP scan position and the G-band reconstruction closest in time is thus around $30 \mathrm{~s}$. A sample of the acquired images is given in Fig. 3.

Because of the importance of a precise co-alignment of all data, we took additional grid target data with all involved instruments. Using these data, we determined the rotation between the TIP spectropolarimetric rasters and the G-band images. The overall shift of these datasets, which also includes the effects of differential refraction, was then derived with sub-arcsec precision using a cross-correlation technique. At the end, we compared the co-spatiality of some G-band bright points with enhanced Stokes $V$ signals as an independent check of the applied co-alignment method.

\section{Emerging loop in observations and inversion}

The temporal evolution of the polarization signals in the small photospheric internetwork area under study, and the magnetic field parameters (i.e., magnetic flux density, field inclination, and field azimuth) derived by the inversion are shown magnified in Fig. 4. The first row displays the speckle-reconstructed G-band images. The superimposed contours mark areas where a significant polarization signal above the noise level was detected. The green contours mark areas of significant net linear polarization. The red and blue contours represent negative and positive circular polarization, respectively, given as $C P=$ $\int(|V| \times$ polarity $) / I_{\mathrm{c}} \mathrm{d} \lambda$.

In the first snapshot taken at 10:37:06 UT, three areas of positive circular polarization are visible. These elements represent the preexisting magnetic field seen in the data set well before, during, and after the time period investigated here. The second snapshot (10:38:47 UT) shows a new, predominantly linearly polarized signal at $(x, y)=\left(-2^{\prime \prime}, 2^{\prime \prime}\right)$, emerging at the location of a granule. In the next minutes, the topology of this feature changes significantly. It expands rapidly, but mostly within the granular boundaries. The next snapshots taken at 10:42:10 UT and 10:43:53 UT already show areas of opposite circular polarization signal occurring symmetrically on both sides of the region with enhanced linear polarization signal. The linear and circular polarization patches partly overlap at these moments. This configuration could correspond to a small magnetic loop-like structure whose footpoints are co-spatial to the areas of opposite circular polarization. The distance of the potential footpoints is roughly $1-1.5^{\prime \prime}$. A slight downward deformation of the preexisting positive circular polarization patch at $\left(-2^{\prime \prime}, 4^{\prime \prime}\right)$, clearly already visible at 10:43:53 UT, suggests the onset of an interaction of the emerging negative polarization patch with this feature. Later on, at 10:45:33 UT, the sudden disappearance of the linear polarization signal is followed by a weakening of both circular polarization patches which have a distance of about $2^{\prime \prime}$ at this moment. Finally, at 10:47:15 UT and subsequently at 10:48:57 UT, the remnants of the circularly polarized features have disappeared completely, and there is no further evidence of their re-occurrence later on in the data set. The whole lifetime of the detected magnetic feature thus was less than $12 \mathrm{~min}$.

The overall course of the event seems to be rather asymmetric in time. Figure 4 indicates a gradual growth of the area of enhanced net linear polarization and the polarization amplitude itself in the four-panel sequence between 10:38:47 UT and 10:43:53 UT, followed by a sudden disappearance in the next panel. The flux emergence leaves no detectable brightness patterns on the underlying granule, which grows steadily in size. The emergent magnetic flux also does not influence the 

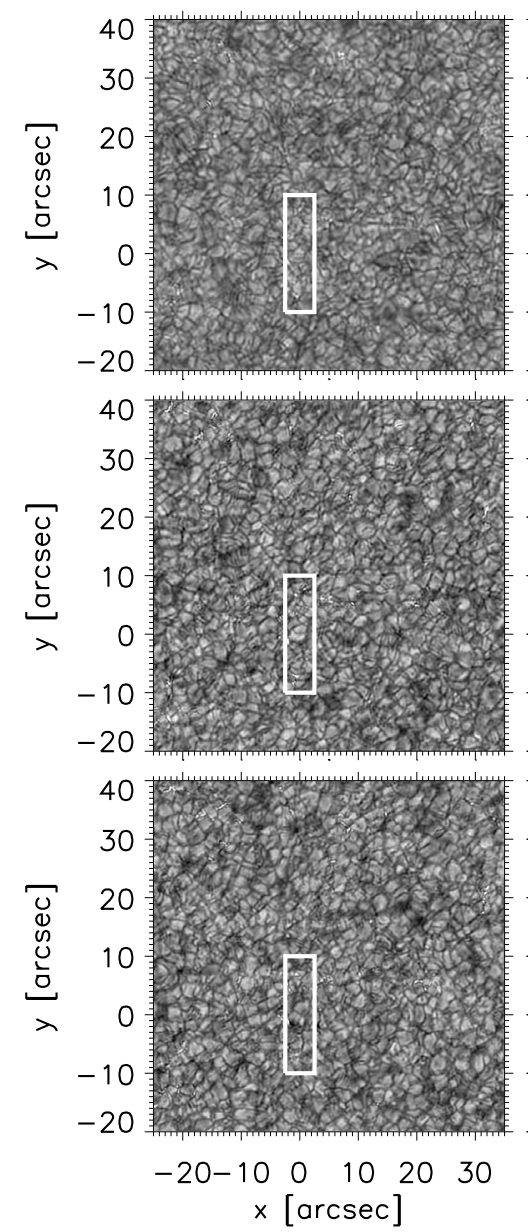
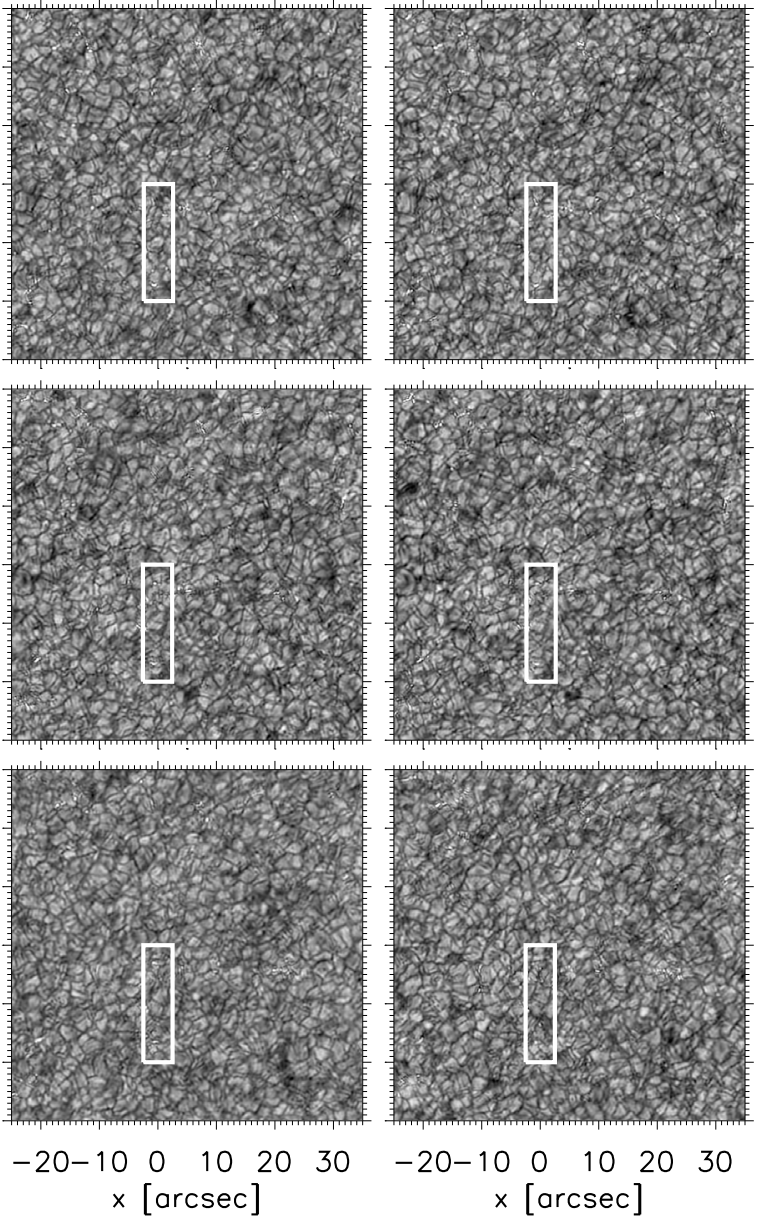
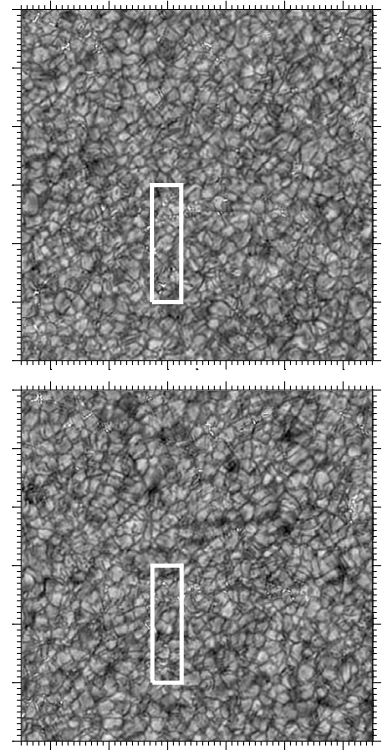

$-20-10001020 \quad 30$

$\times[\operatorname{arcsec}]$

Fig. 3. Sample of the speckle-reconstructed G-band images showing the surroundings of the observed region. The locations of the TIP scans shown in Fig. 1 are marked by white rectangles. The zero point of the coordinate system is shifted 55 arcsec from the disk center.

appearance and brightness of G-band bright points in the vicinity of the granule significantly; most of them stay visible at about the same location for the whole time span of the flux emergence.

The second row of Fig. 4 shows the temporal evolution of the magnetic flux density. Compared with the preexisting ambient magnetism of $\mathrm{kG}$ strength, the flux density of the emerging magnetic feature is much weaker and reaches a mean value of about $450 \mathrm{G}$ throughout its 12 -min period of occurrence. The temporal evolution of this parameter on spatial cuts connecting the two footpoints is also shown in the top panel of Fig. 5. The displayed error bars correspond to the error estimate of the SIR code. Moreover, using the inversion results on the magnetic flux density and the magnetic filling fraction, we estimated the total magnetic flux seen in each of the two footpoints to be around $3 \times 10^{17} \mathrm{Mx}$. However, the inferred value is very probably affected by the limited spatial resolution of the TIP instrument. Therefore, the calculated total magnetic flux likely represents only a lower limit.

The third row of Fig. 4 displays the temporal evolution of the magnetic field inclination which indicates significant differences between the preexisting ambient field and the emerging one. While the former is vertical and stays almost constant in time, the latter shows a much more complex evolution. In its early stages, the inclination angles are close to $90^{\circ}$, corresponding to a horizontal magnetic field (see also the bottom panel of Fig. 5). Later on, the inclination on the edges of the feature becomes more vertical. At 10:43:53 UT, the emerging feature has a clear loop-like structure with two opposite, almost vertical polarities connected by the horizontal field. The trend for a decrease of the inclination continues even after the linear polarization signal has vanished; the remaining tracers of the loop footpoints become still more vertical and also separate further (see also the lower panel of Fig. 5). In the final stage, the upper leg meets with a preexisting magnetic structure of opposite polarity. Reconnection of these two magnetic elements may thus explain the fast disappearance of the polarization signal at this location.

The bottom row of Fig. 4 shows the magnetic azimuth angle. The emerging magnetic structure has an almost constant azimuth of $\sim 75^{\circ}$ during the whole time of its occurrence that is consistent with a rigid magnetic dipole. This again supports the proposed model of a magnetic loop. Due to the $180^{\circ}$ azimuth ambiguity, we cannot resolve without additional information whether the loop has an $\cap$-like shape and is emerging, or an $\cup$-like shape and is submerging (CE07).

To resolve the mentioned ambiguity between an emerging or submerging structure, we analysed the Doppler shifts of the magnetic inversion component. The most relevant maps, showing the phase of the appearance of the structure under study, are displayed in Fig. 6. We found that the middle part of the appearing structure was blueshifted all the time of its occurrence; the velocity reached up to $-2 \mathrm{~km} \mathrm{~s}^{-1}$. This suggests an emergence of a magnetic loop from the lower atmospheric layers. Only later, when the footpoints of the loop become clearly visible, we could detect redshifts at the locations of the footpoints, corresponding to downflows of up to $+2 \mathrm{~km} \mathrm{~s}^{-1}$. This could signify that the material in the loop is draining down along the field lines, which 
P. Gömöry et al.: Magnetic loop emergence within a granule
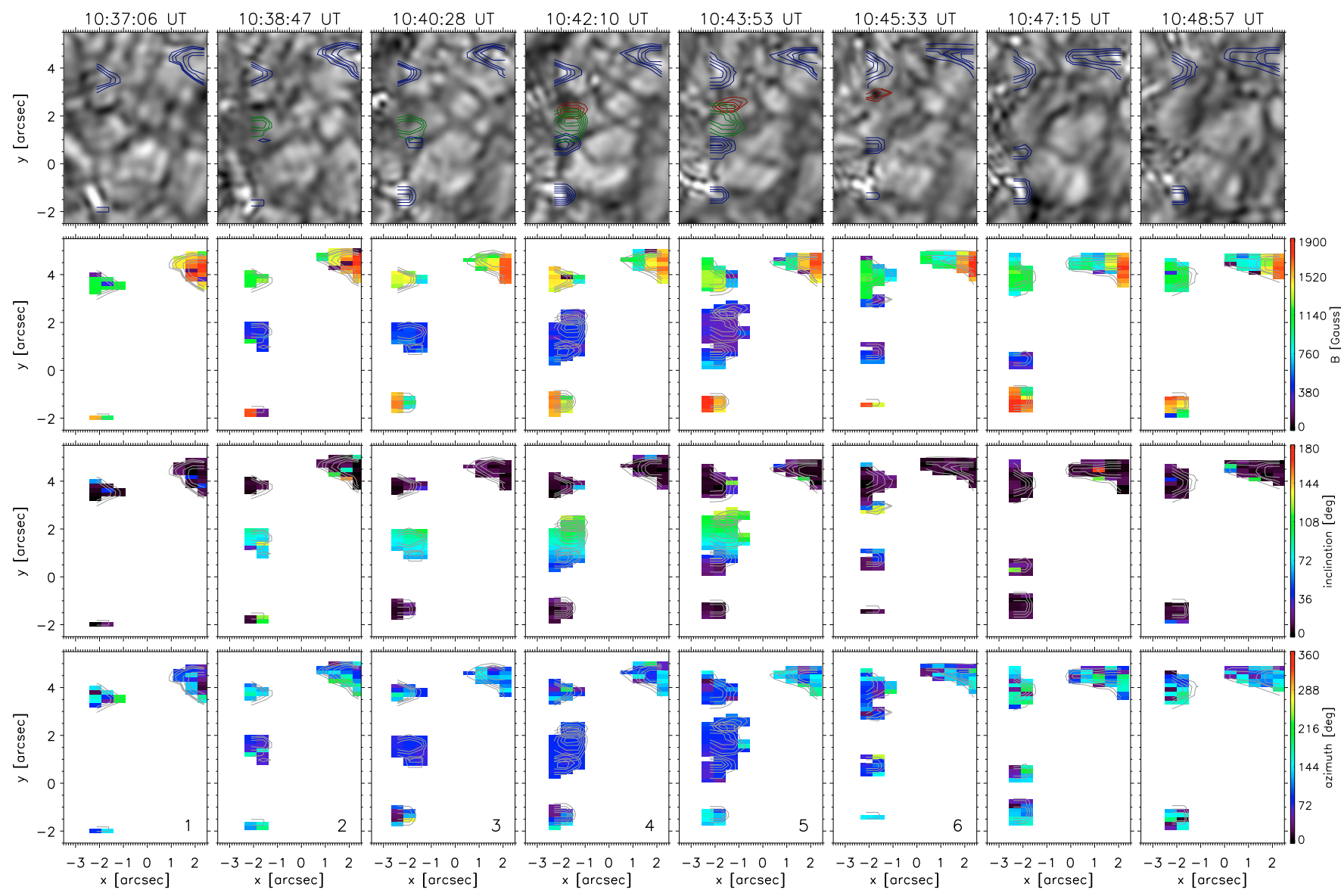

Fig. 4. Temporal evolution during the flux emergence. The G-band images are shown in the 1st row. Areas with enhanced net linear polarization are marked by green contours. The blue and red contours represent positive and negative circular polarization, respectively. The outer contours enclose regions where linear and circular polarization are greater than $0.13 \%$ and $0.11 \%$, respectively, and the innermost contours represent a $0.16 \%$ level for both kinds of polarization. The other rows show the magnetic flux density (2nd row), inclination (3rd row), and azimuth (4th row). The gray contours mark the same polarization levels as before. The times given on top correspond to moments when TIP passed across the occurring feature during the scanning.

is in agreement with the generic loop emergence scenarios. The surrounding magnetic structures are on the other hand predominantly redshifted during the whole displayed time interval.

Based on the observational findings discussed above, we conclude that the temporal evolution, the appearance of the linear and circular polarization signal, and the corresponding parameters of the magnetic field together with the "magnetic" Doppler shifts derived by the inversion all suggest that the observed flux emergence takes place in the shape of a small loop that rises through the photosphere.

\section{Emerging loop in semiempirical simulation}

\subsection{Geometrical model}

To investigate whether the observed event can be explained by flux emergence in the shape of a rising loop, we modeled an $\cap$ shaped loop with the basic geometric parameters as in the observations, and synthesized spectra of the IR lines for it. The loop was placed in a 3D box of around $3000 \mathrm{~km} \times 1000 \mathrm{~km} \times 500 \mathrm{~km}$, with a spatial sampling of $29 \mathrm{~km}$ in $x$ and $y$, and $1 \mathrm{~km}$ in the $z$ direction.

The loop parameters derived from the observations were a horizontal separation speed of its footpoints of $2 \mathrm{~km} \mathrm{~s}^{-1}$, a horizontal extent of around $600 \mathrm{~km}$ at the beginning, and a life time of around $600 \mathrm{~s}$, sampled on 6 maps of the repeated scanning. We then created a loop connecting two footpoints with a distance of $L(k)=600 \mathrm{~km}+k \times 180 \mathrm{~km}(k=1,2, \ldots, 6)$ by prescribing a 2nd order polynomial of the shape

$z(x)=h_{\mathrm{apex}}-\frac{h_{\mathrm{apex}}}{(\alpha \times L / 2)^{2}} x^{2}$,

where $\alpha=0.6$ and $h_{\text {apex }}$ is the location of the apex of the loop. The value of $\alpha$ influences the shape of the loop by stretching or compressing it. It was also used to take into account that the height scale considered for the later conversion to optical depth extended beyond $0 \mathrm{~km}$ to $z=-86.2 \mathrm{~km}$, whereas the crosssection seen in the spectra rather comes from $z>0 \mathrm{~km}$. The loop parameter $h_{\text {apex }}$ was determined by trial-and-error to match the temporal evolution in the observations to $h_{\text {apex }}(k)=-65 \mathrm{~km}$ $+k \times 100 \mathrm{~km}$, corresponding to a rise speed of around $1 \mathrm{~km} \mathrm{~s}^{-1}$.

Figure 7 shows the resulting shape for the central axis of the loop on the six steps on the top. The inclination of the magnetic field to a vertical LOS (2nd panel) was taken from the derivative of the location of the central axis. We determined an appropriate LOS velocity of the material inside the loop from two different contributions, the rise of the loop itself (blueshift in the middle) and a downflow along the field lines (redshift at the footpoints).

The velocity component induced by the rise of the loop was determined from the vertical motion of the loop as defined by Eq. (1). We parametrized the loop for every step $k$ by equidistant 


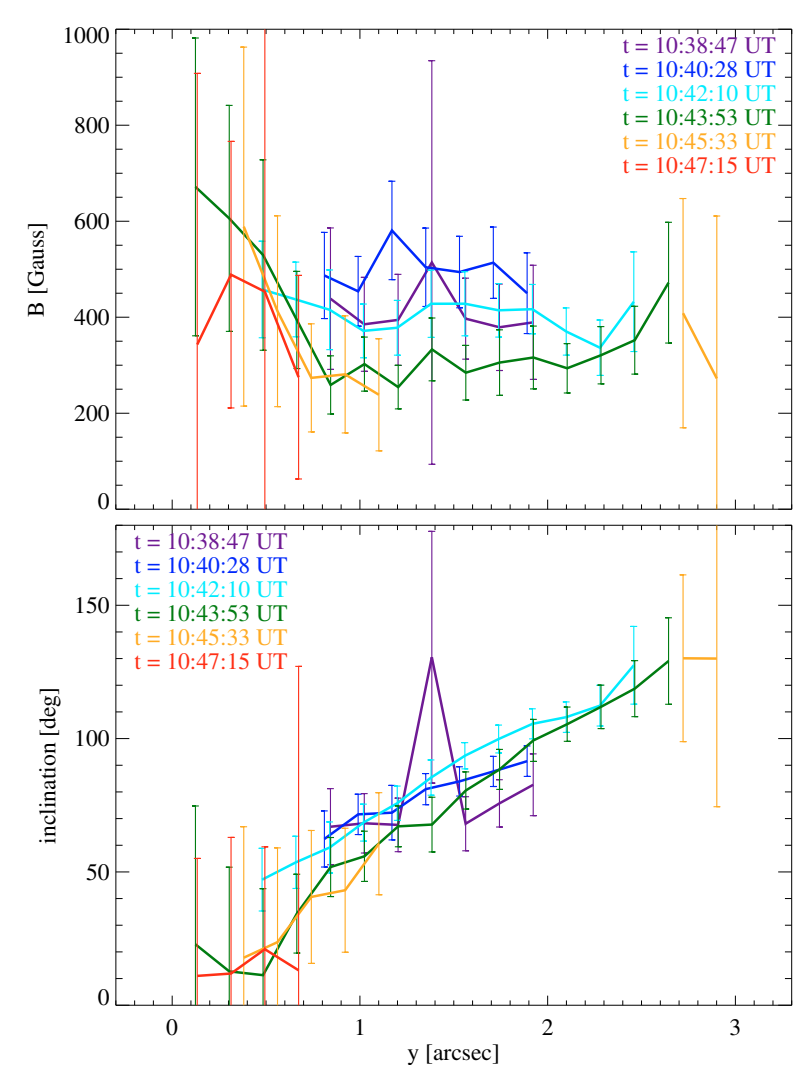

Fig. 5. Cuts along the emerging feature showing the temporal evolution of its magnetic flux density (top) and inclination (bottom).

pieces $l_{i}$ of length $L=1 \mathrm{~km}$ along its axis $\left(i=0 \ldots n_{\max }\right.$, where $n_{\max }$ was set by where the loop reached $\left.z=-1000 \mathrm{~km}\right)$, starting at the apex at each step, to obtain the positions $x\left(k, l_{i}\right), z\left(k, l_{i}\right)$ in the $x-z$ plane (see Fig. 8). The velocity component for a vertical LOS at the position $x\left(k+1, l_{i}\right)$ on the abscissa was then determined as $v_{z}\left(x\left(k+1, l_{i}\right)\right)=\left(z\left(k+1, l_{i}\right)-z\left(k, l_{i}\right)\right) / \mathrm{d} t$, where $\mathrm{d} t$ corresponds to the $\sim 100 \mathrm{~s}$ cadence of the TIP maps. To obtain $v_{z}$ for the evenly spaced grid points used in the spectral synthesis, the velocity values $v_{z}\left(x\left(k+1, l_{i}\right)\right)$ were then interpolated to equidistant $29 \mathrm{~km}$-spacing in $x$. The velocity ranges from $-1 \mathrm{~km} \mathrm{~s}^{-1}$ at the apex to slightly higher velocities of about $-1.4 \mathrm{~km} \mathrm{~s}^{-1}$ near the footpoints due to the additional lateral motion (see $3 \mathrm{rd}$ panel of Fig. 7). For the downflow along the field lines, we assumed a steady flow in free-fall conditions along the loop geometry of step $k=6$, similar to Mein et al. (1996) or Georgakilas et al. (1990, their Eq. (11)). The free-fall condition, however, produces supersonic flows of $v \gg 7 \mathrm{~km} \mathrm{~s}^{-1}$ near the footpoints. We thus have set a limit for the maximum allowed absolute velocity of $4 \mathrm{~km} \mathrm{~s}^{-1}$, which leads to around $+2 \mathrm{~km} \mathrm{~s}^{-1}$ vertical velocity near the footpoints (bottom panel of Fig. 7).

\subsection{Magnetic and thermodynamical properties}

We modeled the loop as a circular flux tube around the central axis by using a field strength $B$ with

$B(r)=500 \mathrm{G} \times \exp \left(\frac{-r^{2}}{2 \sigma^{2}}\right)$,

where $r$ measures the distance from the central axis and $\sigma$ was set to $100 \mathrm{~km}$.

This yields a flux tube with around $240 \mathrm{~km} F W H M$ and an effective radius of $300 \mathrm{~km}$, at which distance the field strength
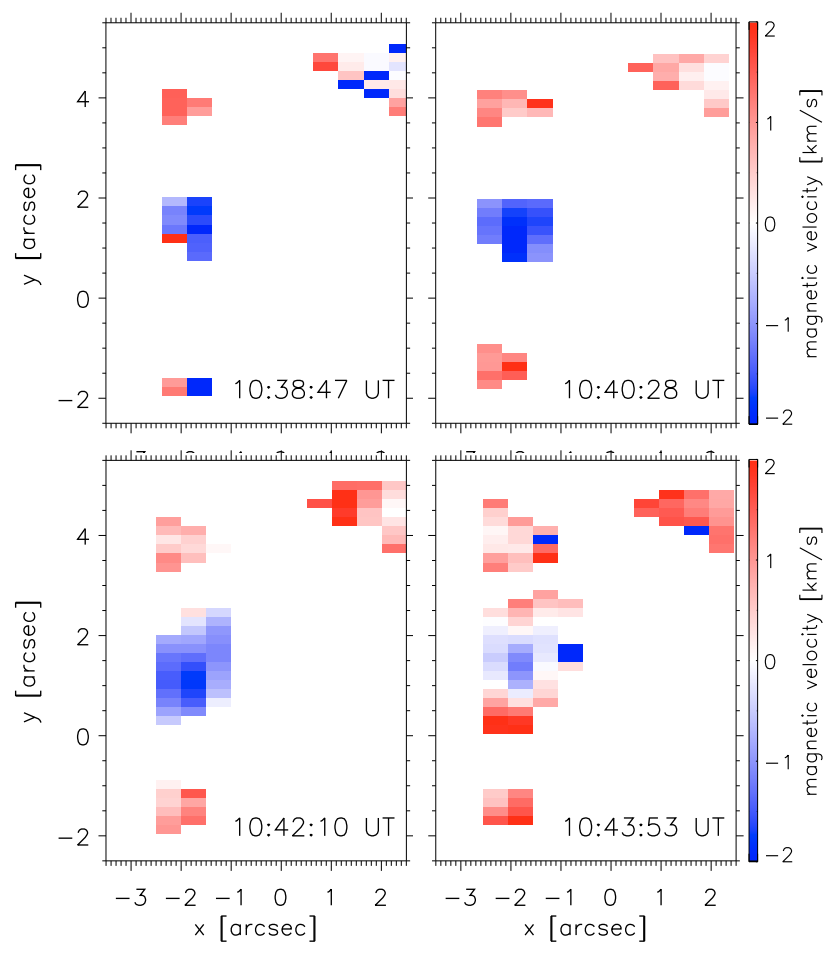

Fig. 6. 2D maps showing temporal evolution of the "magnetic" Doppler shifts. The displayed values are cut at $\pm 2 \mathrm{~km} \mathrm{~s}^{-1}$.

drops to virtually zero. Integration of Eq. (2) over the radius yields a total magnetic flux of around $2.7 \times 10^{17} \mathrm{Mx}$, which is slightly below the magnetic flux value derived from the inversion results. Figure 9 displays the final geometry by showing isosurfaces of $100 \mathrm{G}$ field strength for the six steps of the flux emergence. At the first step, most of the loop is below the formation height of the spectral lines considered, and thus induces only a small polarization signal in the spectra.

We used the tabulated values of the HSRA model (Gingerich et al. 1971) to define the thermodynamic properties like temperature, gas density, or electron pressure needed for the spectral synthesis. We considered an optical depth range from $\log \tau=+1.4$ to around -3 , which covers the formation heights of the IR lines at 1.56 micron (Cabrera Solana et al. 2005). We did not consider the effect of the presence of the loop on the stratifications (or vice versa) by enforcing hydrostatic equilibrium.

To determine the magnetic field stratifications for a vertical LOS from the 3D topology, we used cuts through the 3D box along the $z$-axis. The field strength $B(x, y, z)$ can then be derived from the distance of the point $(x, y, z)$ from the closest piece of the central axis using Eq. (2). The stratifications of $B$ can differ strongly from a Gaussian shape, depending on where along the loop the cut is located; near the footpoints, one obtains a Gaussian shape stretched in $z$, whereas close to the apex the Gaussian shape is preserved (Fig. 10).

\subsection{Spectral synthesis and degradation of spatial resolution}

Using the derived stratifications for the thermodynamic variables and the magnetic field strength, we then synthesized spectra of the three spectral lines Fe I $1564.8 \mathrm{~nm}$, a weak blend of Fe I at $1564.7 \mathrm{~nm}$, and Fe I $1565.2 \mathrm{~nm}$ with the SIR code. The simulated spectra differ strongly from the observations in spatial resolution. To improve the match to the observations, we degraded the spatial resolution by a convolution with a 2D Gaussian kernel 

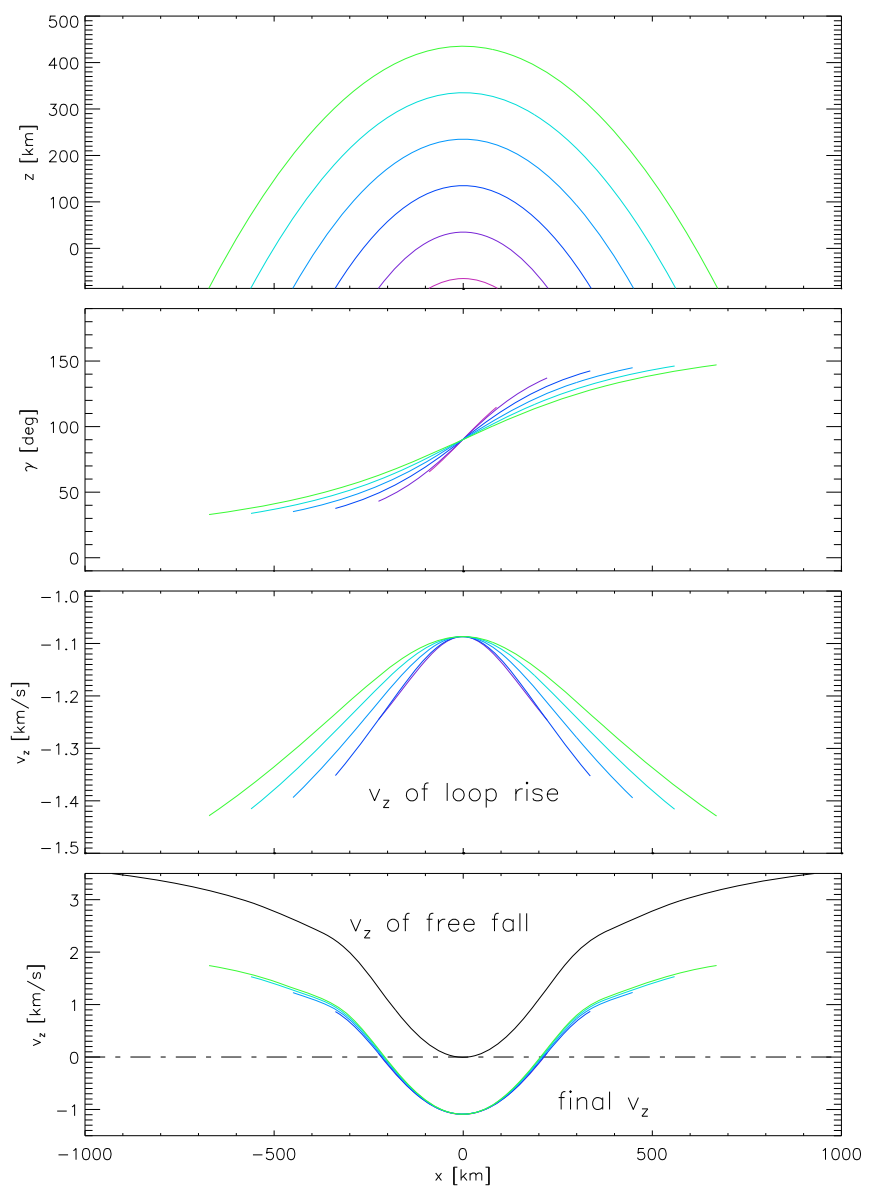

Fig. 7. Temporal development of the simulated loop in six time steps. From top to bottom: Central loop axis, magnetic field inclination, LOS velocity component $v_{z}$ of the loop rise, free fall, and their addition to the final $v_{z}$.

of $0.56 F W H M$, roughly corresponding to the diffraction limit of the VTT at IR wavelengths. We then calculated 2D maps of total linear and circular polarization $L$ and $|V|$, respectively, and the polarity of the Stokes $V$ signal analogously to the observations.

Figure 11 shows these maps for both the original and the spatially degraded simulation, and the corresponding section from the observation, for a visualization of the effects of spatial resolution and spatial sampling. The display ranges of each quantity in Fig. 11 are given in Table 1. For the observations, the value of the wavelength-integrated quantities cannot reach zero because of the noise. With the assumption that the lowermost value in the observations corresponds to a pure noise signal, the dynamic range of total linear polarization $L$ in the observations matches fairly well that of the convolved simulation, whereas for total circular polarization $|V|$ it is significantly smaller. The spatial smearing is sufficient to reduce the circular polarization signal in the first step to values below the threshold used for the observations (compare $1 \mathrm{st}, 7 \mathrm{th}$, and 13th column). The elongated loop structure seen in $L$ of the full-resolution spectra (top left) is lost after the spatial degradation; the strong linear polarization signal appears there in the shape of an only slightly elongated patch. The two polarities in the degraded synthetic polarity map are seen to touch each other persistently up to step 3 , similar to the observed behavior. The effect of applying the Gaussian kernel is to first order already equivalent to sampling the degraded data with the pixel size of the observations (white rectangle in the bottom right panel, 0 ' $^{\prime} 45 \times 00^{\prime} 17$ pixel). We thus skipped an

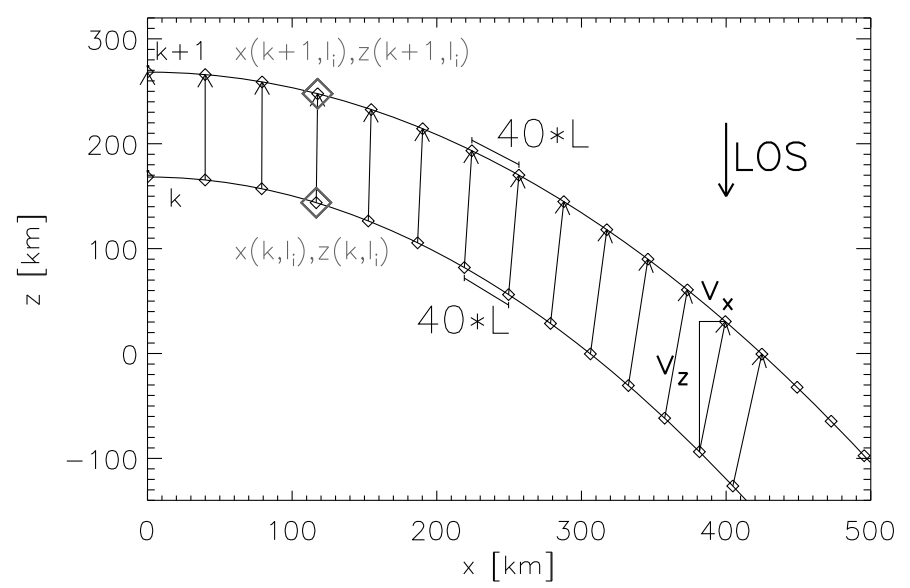

Fig. 8. Calculation of velocity components $\left(v_{x}, v_{z}\right)$ from the location of the central axis in the steps $k$ and $k+1$.

additional re-sampling with the TIP pixel size for the simulation spectra shown in the following.

\subsection{Direct comparison of observed and synthetic spectra}

For a direct comparison of observed and synthetic spectra, we took cuts along the central axis of the feature in the simulation. For the observations, we chose the slit spectra of a scan step near the beginning of each repeated map, since the structure is roughly oriented along the slit. Figure 12 shows the IQUV spectra for these six cuts. The observed spectra (at bottom) show some features that are naturally missing in the simulation (at top): the intensity and velocity pattern of the granulation, and additional polarization signal at the upper end of the cut corresponding to the preexisting patch of network fields $\left(y \sim 4^{\prime \prime}\right)$. For a comparison of the polarization level in the simulation and observation, we added an rms noise of $1 \times 10^{-3}$ to the simulation spectra of Stokes $Q$ of the $1565.2 \mathrm{~nm}$ line and Stokes $U$ of the $1564.8 \mathrm{~nm}$ line in the first three steps (white rectangle at top middle). The noise level is sufficient to suppress the polarization signal of the less Zeeman-sensitive line at $1565.2 \mathrm{~nm}$ as in the observed spectra. The maximal linear polarization amplitudes of the simulation and observation also roughly match (up to $1.5 \%$ of $I_{\mathrm{c}}$ for $Q$ and $U$ ). The Stokes $V$ amplitude of the simulation exceeds the observations for the steps 5 and 6 , where in the observations the cancellation with the preexisting network patch may already have happened. The observed trend of polarization signal with time is reproduced in the simulation: a monotonical increase of $V$ amplitude until step 5, increase and decrease for $Q$ and $U$ with maximal amplitude at step 4 . The observed spectra still show a faint $Q$ and $U$ signal in step 6, only seen in the $1564.8 \mathrm{~nm}$ line. Near the footpoints, the observed Stokes $V$ signal has a drop-like shape, with a decrease of the splitting and a displacement to the blue when moving towards the opposite polarity that also shows up in the simulation spectra. The observed Stokes $Q$ signal in step 3 has the same curved shape in $y$ as the synthetic spectra of steps 3 and 4 , indicating the blueshift in the middle and the redshift near the footpoints.

At step 4 (10:42:10 UT), the loop-like structure is seen most clearly in the observations (cf. Figs. 4 or 11). We thus selected the profiles at the center of the loop and both footpoints of this step from observations and the simulation (short horizontal bars in Fig. 12) for the direct comparison shown in Fig. 13. Besides the amplitude of Stokes $V$, the continuum intensity level and 

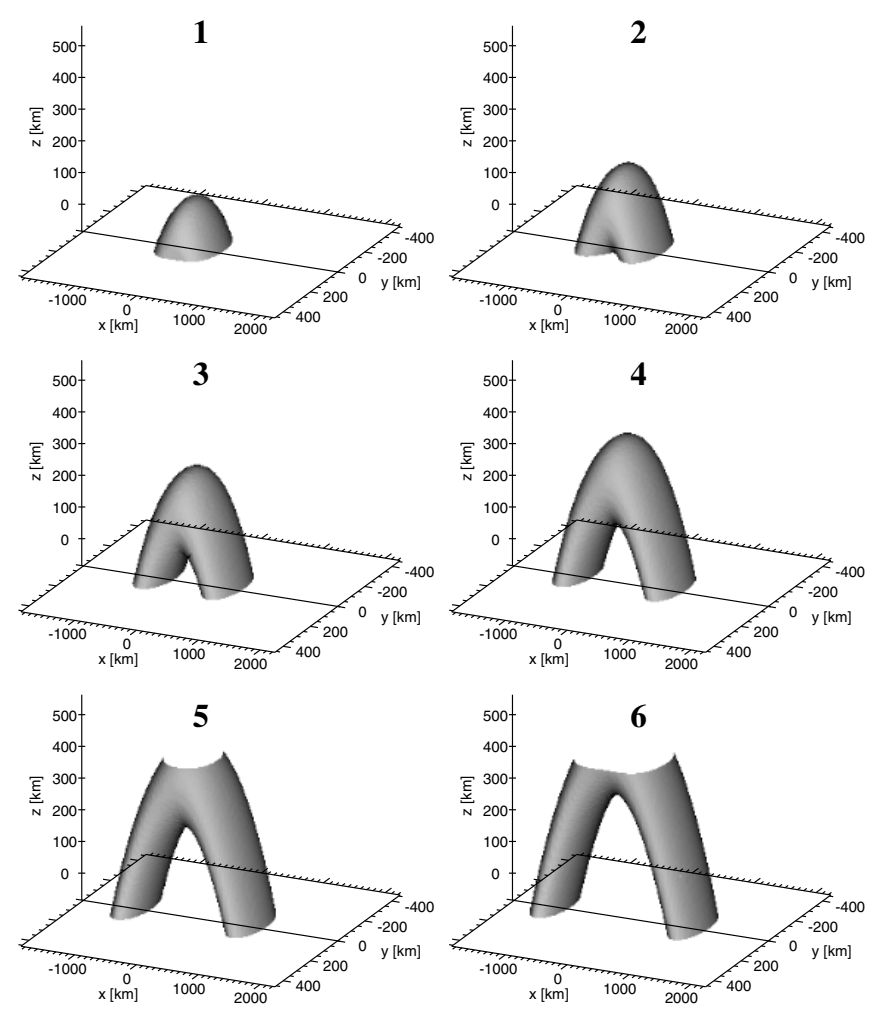

Fig. 9. Magnetic field topology during the flux emergence simulation.

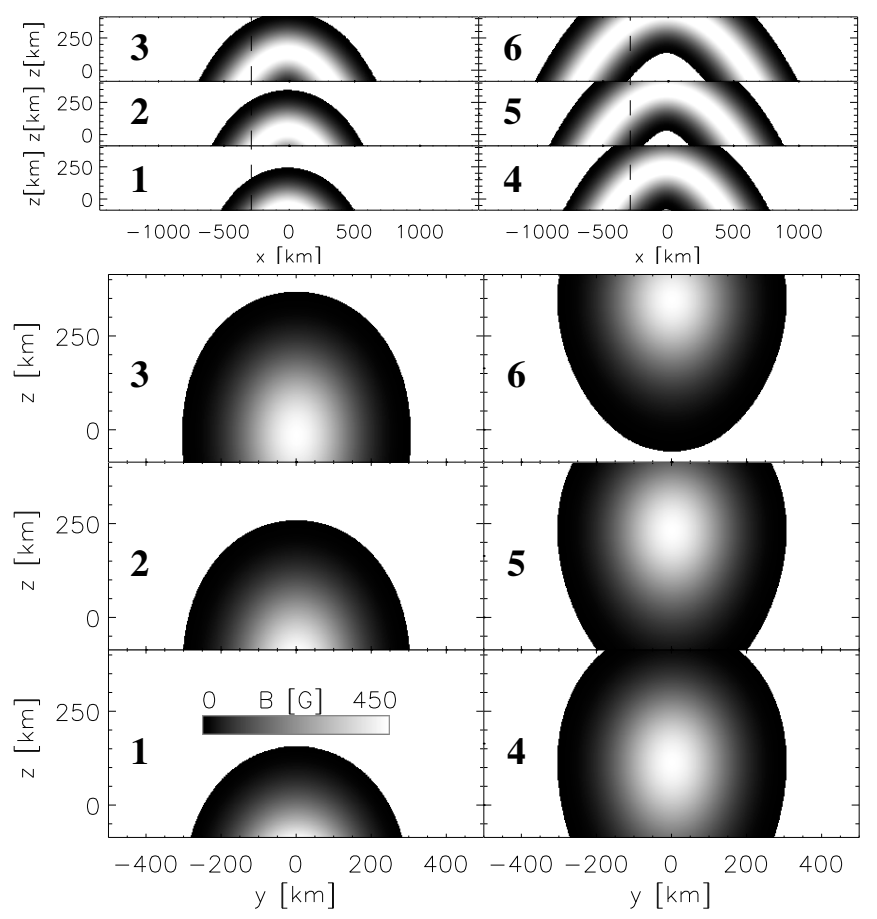

Fig. 10. Magnetic field strength in side view in the $x-z$ plane (top), and cut in the $y-z$ plane across one of the footpoints (bottom). The location of the cut is marked by a dash-dotted vertical line in the upper plot.

the Doppler shift in the lower footpoint, the match is surprisingly good, despite the simplifications used in generating the geometric model. We thus conclude that the observed spectra are in good agreement with a rising magnetic flux tube of around

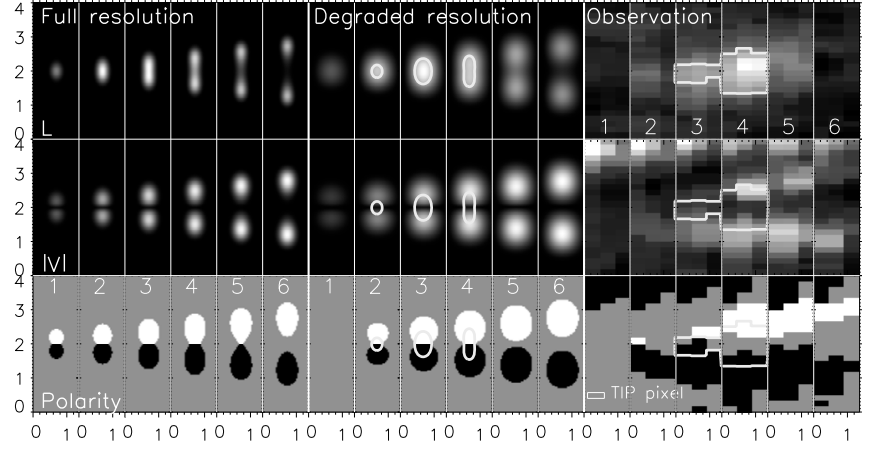

Fig. 11. Top to bottom: total linear polarization, circular polarization, and polarity. Left: full resolution of the simulation. Middle: after degradation. Right: corresponding section of the FoV in the observations. The white rectangle in the bottom right panel indicates the size of a pixel in the observations. Contour lines trace strong linear polarization signal. Each panel and quantity is displayed with an individual range for better visibility (see text for details). Tickmarks are in arcsec.

Table 1. Display ranges for Fig. 11.

\begin{tabular}{lccc}
\hline \hline & $\begin{array}{c}\text { Simulation at } \\
\text { full resolution }\end{array}$ & $\begin{array}{c}\text { Convolved } \\
\text { simulation }\end{array}$ & $\begin{array}{c}\text { Observations / dynamical } \\
\text { range }\end{array}$ \\
\hline$L$ & $0-1.18$ & $0-0.4$ & $1.23-1.71 / 0-0.48$ \\
$|V|$ & $0-4.1$ & $0-1.48$ & $0.9-1.6 / 0-0.7$ \\
\hline
\end{tabular}

$250 \mathrm{~km}$ diameter, $3 \times 10^{17} \mathrm{Mx}$ magnetic flux, and a rise speed of $1 \mathrm{~km} \mathrm{~s}^{-1}$.

\section{Discussion and conclusions}

High-resolution surveys of large areas of quiet-Sun internetwork (Lites et al. 2008) and plage regions (Ishikawa et al. 2008) revealed widespread occurrences of relatively strong horizontal fields over granules, accompanied by vertical bipolar fields cospatial with up- and downflows (Martínez González et al. 2007). On the other hand, individual granules are often collocated with strong unipolar fields decoupled from granular outflows (OR08, Bello González et al. 2008).

CE07 reported for the first time the emergence of a magnetic loop inside of a granule whose footpoints drifted towards intergranular lanes. Here we present new observational evidence for a similar event, whose onset manifests itself through enhanced net linear polarization within an evolved granule. Soon after, a pair of opposite circularly polarized patches appear symmetrically on each side of the linear polarization forming a small magnetic dipole bridged by a horizontal magnetic field. As long as both patches of circular polarization are visible, they are about of the same size and show a balanced magnetic flux of opposite sign $\left(+2.9\right.$ and $-3.1 \times 10^{17} \mathrm{Mx}$ in step 4). This supports the conclusion made by Lamb et al. (2008) that the model of an asymmetric dipole emergence is very unlikely.

The fast disappearance of the linear polarization signal after 10:43:53 UT (step 5, see Figs. 1, 4, or 11) is in striking contrast with its gradual growth seen from 10:38:47 UT to 10:43:53 UT. Can we understand it just by an upward extending loop reaching up to a higher atmospheric layer undetectable by the Fe I lines as suggested in CE07? If we consider the loop top as a magnetic perturbation propagating upwards through otherwise non-magnetic layers, then a smooth decay of contribution and response functions with height would imply slower ceasing of 


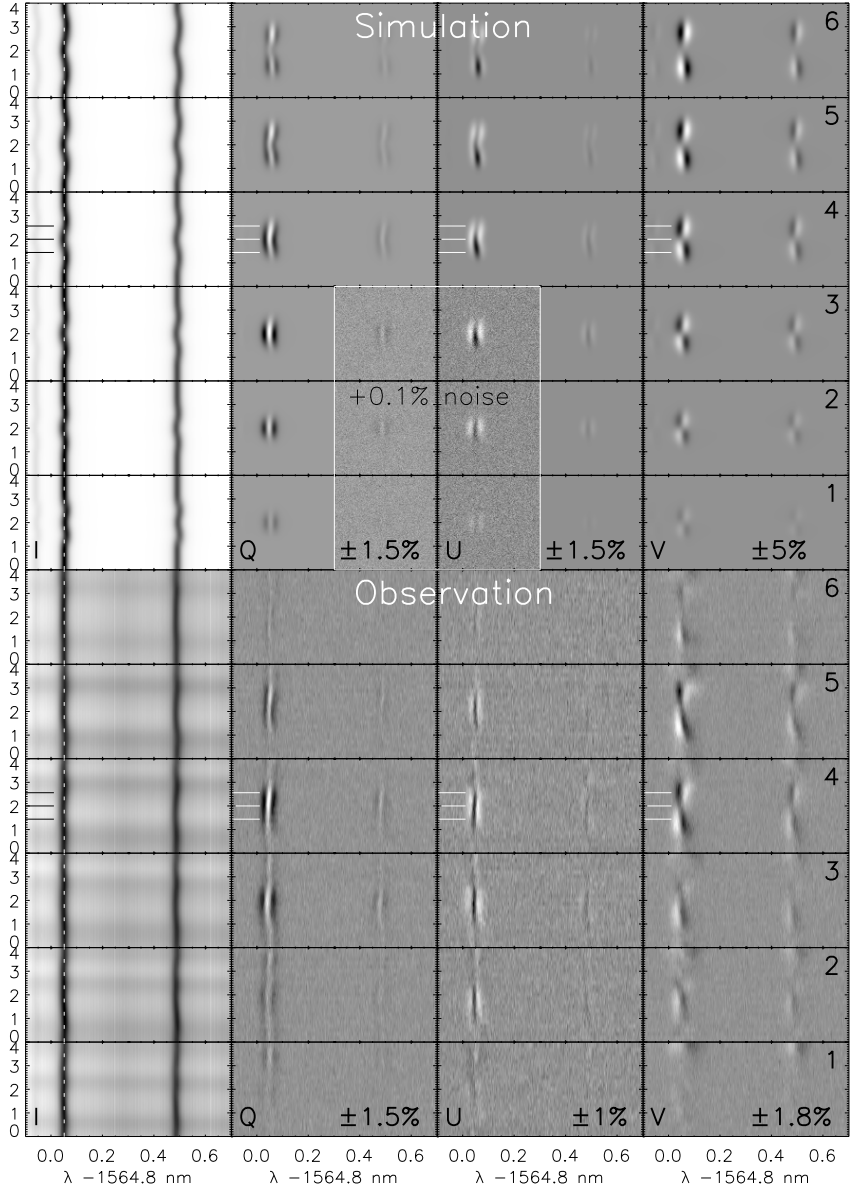

Fig. 12. Comparison of observed (bottom) and simulated spectra (top). Left to right: Stokes IQUV. Bottom to top: time steps 1 to 6 . The display ranges for $Q U V$ are given at bottom right in each panel. The vertical dashed line in Stokes $I$ denotes the rest wavelength of the $1564.8 \mathrm{~nm}$ line. The short horizontal bars at the left in IQUV of step 4 denote the location of the profiles shown in Fig. 13.

the net linear polarization, perhaps detectable on the next frame. Since the contribution and response functions of Stokes $Q$ and $U$ to magnetic perturbations can be very complex (del Toro Iniesta 2003), their detailed computations would help to understand the disappearance of linear polarization after 10:43:53 UT. But after 10:43:53 UT the circular polarization signal of the footpoint that meets an opposite-polarity magnetic field also weakens rapidly. This could indicate magnetic reconnection between the emergent flux and the preexisting magnetic fields. If reconnection thus is such a common feature, it could explain why OR08 found no trace of the flux emergence in the chromospheric layers.

The disappearance of the polarization signal could, however, also be related to other causes. In the rising loop simulation, the linear polarization amplitude at the last step is again comparable to the first one. Taking into account the noise present in the observations, the polarization signal could also be simply reduced below the detection limit. Another possibility could be a slight spatial drift of the feature with time that would remove it partly out of the observed FoV, because it already was located only in the first three steps of each repeated scan (see Fig. 1). Moreover, the possible expansion of the magnetic loop with height (and time) could also affect the measurement of the linear signal above the detection threshold. From the comparison with the simulated loop, we conclude, however, that at least the

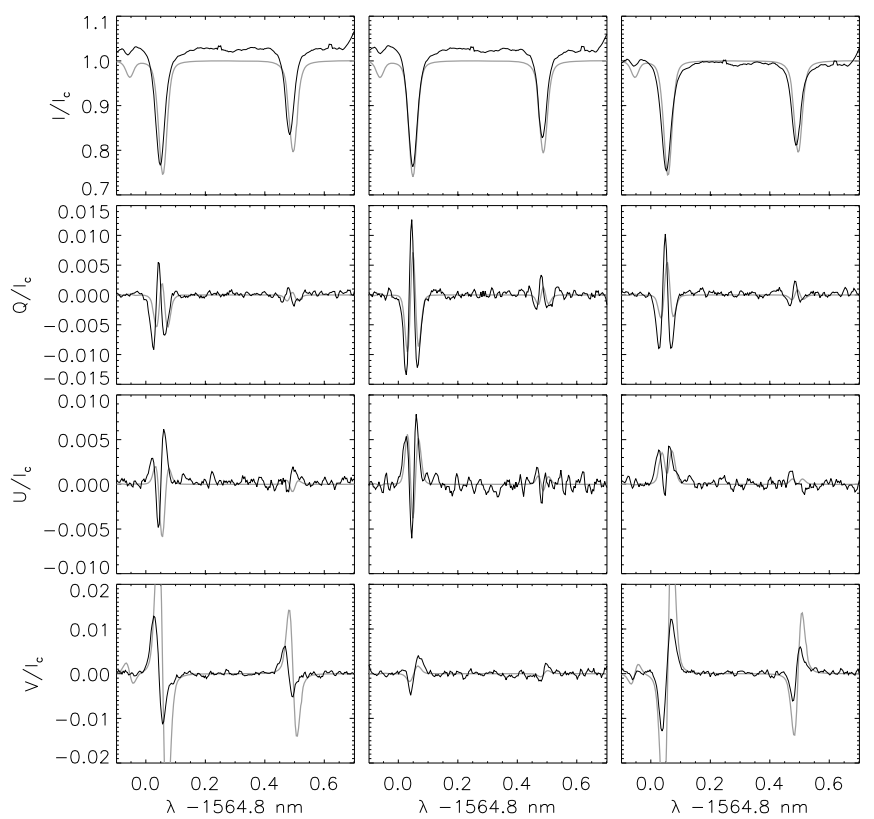

Fig. 13. Comparison of observed (black) and synthetic spectra (gray) in step 4. Top to bottom: Stokes IQUV. Left to right: Lower footpoint, center of loop, upper footpoint.

ascent until maximal linear polarization signal is reached is fully traced by the observations.

Our flux density estimate of $\sim 450 \mathrm{G}$ found in the emerging loop is comparable to the values reported in Martínez González et al. (2007) and Ishikawa et al. (2008) for small-scale magnetic loops in the solar internetwork and plage region, respectively; it significantly exceeds, however, the $10-100 \mathrm{G}$ given by Martínez González \& Bellot Rubio (2009). This could be due to the assumption of a magnetic filling factor of unity by the latter authors. The loop footpoints separate with time by around $2 \mathrm{~km} \mathrm{~s}^{-1}$, drifting in opposite directions towards intergranular lanes. Moreover, our estimate of the magnetic flux density shows that the field is stronger than the typical equipartition value of 400 G (Cheung et al. 2007; Ishikawa et al. 2008). This suggests that both granular outflow and relaxation of the magnetic tension of the loop may drive the footpoints.

How does our loop compare to the one reported in CE07? They occupy similar locations and enclose too little magnetic flux to leave any trace on the underlying granules or ambient Gband bright points. On the other hand, our loop displays a higher magnetic flux density and a longer lifetime.

Another difference to the loop reported in CE07 is that in our case one loop footpoint meets the ambient field of opposite polarity, which is followed by the fast disappearance of the loop, in contrast to its more gradual emergence. This is very symptomatic of field cancellation by magnetic reconnection, as reported in Cheung et al. (2008) and Guglielmino et al. (2008). The magnetic reconnection likely drags loop remnants very rapidly out of the line-forming domain less than 12 min after the first trace of the loop top was detected.

The magnetic loop emergence presented here is probably just a particular event in a multitude of similar ones occurring continuously in the solar internetwork. Simulations of small-scale magnetoconvection by, e.g., Stein \& Nordlund (2006) show examples of granular-sized magnetic loops with similar behavior. They probably build up a sub-canopy of horizontal fields over granules (Wedemeyer-Böhm et al. 2009). Surface dynamo simulations by Schüssler \& Vögler (2008) and Steiner et al. (2008) 
clearly show that this new component of the internetwork is a direct consequence of magnetic flux expulsion from the granular interior, not only to the intergranular lanes, but also to the upper photosphere. Cheung et al. $(2007,2008)$ found in numerical simulations that emerging flux regions also contribute to the flux accumulation and its maintenance above granular upflows in the magnetic inversion layer. Our results show one particular example of this local process albeit terminated, probably due to magnetic reconnection. Better temporal and spatial resolution is needed for more detailed information on the process than our observations provide.

Acknowledgements. The VTT is operated by the Kiepenheuer-Institut für Sonnenphysik, Freiburg, at the Observatorio del Teide of the Instituto de Astrofísica de Canarias. The observations have been funded by the OPTICON trans-national access program funded by the European Commissions Sixth Framework Programme. The authors are grateful for the OPTICON support provided. Work of P. G., J. R., A. K., and J. K. was supported partly by the Slovak Research and Development Agency under the contract No. APVV-0066-06. This work also was partly supported by the Deutsche Forschungsgemeinschaft grant No. DFG 436 SLK 113/13/0-1. We thank H.P. Doerr (KIS) for the support with the Speckle computers at the VTT. The authors would like to thank the anonymous referee for helpful comments. This research has made use of NASA's Astrophysics Data System.

\section{References}

Abbett, W. P. 2007, ApJ, 665, 1469

Beck, C., Schlichenmaier, R., Collados, M., Bellot Rubio, L., \& Kentischer, T. J. 2005, A\&A, 443, 1047

Beck, C., Mikurda, K., Bellot Rubio, L. R., Kentischer, T., \& Collados, M. 2007, in Modern solar facilities - advanced solar science, ed. F. Kneer, K. G. Puschmann, \& A. D. Wittmann, Proceedings of a Workshop held at Göttingen, September 27-29, 2006, Göttingen, Germany, Universitätsverlag Göttingen, 55

Bello González, N., Okunev, O., \& Kneer, F. 2008, A\&A, 490, L23

Bellot Rubio, L. R., Balthasar, H., \& Collados, M. 2004, A\&A, 427, 319

Berkefeld, T. 2006, in Modern solar facilities - advanced solar science, ed. F. Kneer, K. G. Puschmann, \& A. D. Wittmann, Proceedings of a Workshop held at Göttingen, September 27-29, 2006, Göttingen, Germany, Universitätsverlag Göttingen, 107

Cabrera Solana, D., Bellot Rubio, L. R., \& del Toro Iniesta, J. C. 2005, A\&A, 439, 687

Cattaneo, F. 1999, ApJ, 515, L39

Centeno, R., Socas-Navarro, H., Lites, B., et al. 2007, ApJ, 666, L137 (CE07)

Cheung, M. C. M., Schüssler, M., \& Moreno-Insertis, F. 2007, A\&A, 467, 703

Cheung, M. C. M., Schüssler, M., Tarbell, T. D., et al. 2008, ApJ, 687, 1373
Collados, M. 1999, in Third Advances in Solar Physics Euroconference: Magnetic Fields and Oscillations, ed. B. Schmieder, A. Hofmann, \& J. Staude, ASP Conf. Ser., 184, 3

Collados, M. 2003, in Polarimetry in Astronomy, ed. S. Fineschi, Proc. SPIE 4843, 55

Collados, M., Lagg, A., Díaz García, J. J., et al. 2007, in The Physics of Chromospheric Plasmas, ed. P. Heinzel, I. Dorotovič, \& R. J. Rutten, Proceedings of the conference held at the University of Coimbra, October 9-13, 2006, Coimbra, Portugal, ASP Conf. Ser., 368, 611

Georgakilas, A. A., Alissandrakis, C. E., \& Zachariadis, T. G. 1990, Solar Phys., 129,277

Gingerich, O., Noyes, R. W., Kalkofen, W., et al. 1971, Solar Phys., 18, 347 Guglielmino, S. L., Zuccarello, F., Romano, P., et al. 2008, ApJ, 688, L111 Ishikawa, R., Tsuneta, S., Ichimoto, K., et al. 2008, A\&A, 481, L25

Kentischer, T. J., Schmidt, W., Sigwarth, M., et al. 1998, A\&A, 340, 569 Khomenko, E., \& Collados, M. 2007, ApJ, 659, 1726

Kosugi, T., Matsuzaki, K., Sakao, T., et al. 2007, Solar Phys., 243, 3

Kučera, A., Beck, C., Gömöry, P., et al. 2008, in Proceedings of the 12th European Solar Physics Meeting, September 8-12, 2008, Freiburg, Germany, 2.52. Available online at http://espm.kis.uni-freiburg.de

Lamb, D. A., DeForest, C. E., Hagenaar, H. J., Parnell, C. E., \& Welsch, B. T. 2008, ApJ, 674, 520

Lites, B. W., Elmore, D. F.,\& Streander, K. V. 2001, in Advanced Solar Polarimetry - Theory, Observation, and Instrumentation, ed. M. Sigwarth, Proceedings of the 20th NSO/Sac Summer Workshop, ASP Conf. Ser., 236, 33

Lites, B. W., Kubo, M., Socas-Navarro, H., et al. 2008, ApJ, 672, 1237

von der Lühe, O., Soltau, D., Berkefeld, T., et al. 2003, Proc. SPIE 4853, ed. by S. L. Keil, \& S. V. Avakyan, 187

Martínez González, M. J., Collados, M., Ruiz Cobo, B., et al. 2007, A\&A, 469, L39

Martínez González, M. J., \& Bellot Rubio, L. R. 2009, ApJ, 700, 1391

Mein, P., Demoulin, P., Mein, N., et al. 1996, A\&A, 305, 343

Orozco Suárez, D., Bellot Rubio, L. R., del Toro Iniesta, J. C., et al. 2008, A\&A, 481, L33 (OR08)

Ruiz Cobo, B., \& del Toro Iniesta, J. C. 1992, ApJ, 398, 375

Schaffenberger, W., Wedemeyer-Böhm, S., Steiner, O., et al. 2006, in Solar MHD Theory and Observations: A High Spatial Resolution Perspective, ed. J. Leibacher, R. F. Stein, \& H. Uitenbroek, Proceedings of the conference held at the National Solar Observatory, July 18-22, 2005, Sacramento Peak, New Mexico, USA, ASP Conf. Ser., 354, 345

Schlichenmaier, R., \& Collados, M. 2002, A\&A, 381, 668

Schröter, E. H., Soltau, D., \& Wiehr, E. 1985, Vist. Astron., 28, 519

Schüssler, M., \& Vögler, A. 2008, A\&A, 481, L5

Stein, R. F., \& Nordlund, A. 2006, ApJ, 642, 1246

Steiner, O., Rezaei, R., Schaffenberger, W., et al. 2008, ApJ, 680, L85

del Toro Iniesta, J. C. 2003, Introduction to Spectropolarimetry (Cambridge, UK: Cambridge University Press)

Tritschler, A., Schmidt, W., Langhans, K., et al. 2002, Solar Phys., 211, 17

Vögler, A., Shelyag, S., Schüssler, M., et al. 2005, A\&A, 429, 335

Wedemeyer-Böhm, S., Lagg, A., \& Nordlund, A. 2009, Space Sci. Rev., 144, 317

Wöger, F., von der Lühe, O., \& Reardon, K. 2008, A\&A, 488, 375 